\title{
L'institutionnalisation des marchés informels à Paris : une situation d'entre-deux dans le gouvernement des espaces publics
}

Hélène Balan

\section{(2) OpenEdition}

Journals

Édition électronique

URL : http://journals.openedition.org/cdg/387

DOI : $10.4000 /$ cdg.387

ISSN : 2107-7266

Éditeur

UMR 245 - CESSMA

Référence électronique

Hélène Balan, "L'institutionnalisation des marchés informels à Paris : une situation d'entre-deux dans le gouvernement des espaces publics », Carnets de géographes [En ligne], 7 | 2014, mis en ligne le 01 décembre 2014, consulté le 02 mai 2019. URL : http://journals.openedition.org/cdg/387 ; DOI : $10.4000 /$ cdg.387

\section{cc) (i) $\odot$}

La revue Carnets de géographes est mise à disposition selon les termes de la Licence Creative Commons Attribution - Pas d'Utilisation Commerciale - Pas de Modification 4.0 International. 
Carnets de géographes, $\mathrm{n}^{\circ} 7$, juin 2014

Rubrique Carnets de recherche

\section{L'institutionnalisation des marchés informels à Paris : une situation d'entre-deux dans le gouvernement des espaces publics}

HELENE BALAN

Doctorante en géographie à l'Université Paris Ouest Nanterre Laboratoire LAVUE-Mosaïques helenebalan@hotmail.fr

\section{Résumé}

A Paris, le développement dans l'espace public de marchés informels pouvant rassembler jusqu'à plusieurs centaines de personnes soulève la question de l'institutionnalisation de l'informel dans une métropole européenne gouvernée par une municipalité socialiste. Cet article s'interroge sur les enjeux territoriaux et scalaires des controverses politiques autour de ces marchés informels. II analyse la situation d'entre-deux dans le gouvernement des espaces publics ouverte par la défense d'un "droit à la ville » de ces vendeurs précaires et d'une gestion locale de ces marchés à travers la mobilisation des biffins, vendeurs d'articles de récupération, d'après un ancien nom des chiffonniers. II s'agit de montrer comment les controverses autour des marchés informels aboutissent à une gestion au cas par cas et à la marge de cette économie de survie urbaine, qui révèle les limites de la promotion du local comme échelle de l'alternative.

\footnotetext{
Abstract

In Paris, the development of informal markets sometimes gathering up to hundreds of people in public space raises the question of the institutionnalization of informal activities in a European metropolis governed by a socialist municipality. This paper questions the territorial and scalar issues of political controversies about these informal markets. It analyses the "in-between" situation created in the governement of public spaces by the defense of a "right to the city" for these precarious vendors and a local management of these markets through the mobilization of the "biffins" - street vendors of recovery goods, after a former name for scavengers. The point is to show how the controversies about informal markets lead to a case-by-case and at the margin treatment of this urban survival economy, which reveals the limites of promoting the local as the right scale for alternative.
} 
A Paris aujourd'hui, des marchés informels se tiennent "à la sauvette " en marge des marchés aux Puces de Saint-Ouen, de Montreuil et de Vanves, ainsi qu'aux carrefours de Belleville et Barbès dans le nord-est parisien (figure 1). Le phénomène s'y est étendu autour de 2008, avec le début de la récession économique. Ces marchés rassemblent de plusieurs dizaines à plusieurs centaines de personnes en situation de précarité vendant majoritairement des articles de récupération et, dans une moindre mesure, des produits neufs. Des marchés similaires se sont également développés, dans de moindres proportions, dans des métropoles de province. C'est le cas par exemple dans la banlieue de Lyon ou à Toulouse, où un encadrement de la vente informelle d'articles de récupération a été mis en place $^{1}$ en 2011.

A Paris, l'extension des marchés informels est survenue parallèlement à la mobilisation de vendeurs d'articles de récupération présents en marge du marché aux Puces de Saint-Ouen. Redéfinis comme biffins, d'après un ancien nom des chiffonniers, ceux-ci se constituent en 2006 en association pour dénoncer la répression dont ils font l'objet, "sur les conseils des élus Verts » (Milliot, $2010: 44$ ) du 18e arrondissement. En 2009, ils obtiennent des pouvoirs municipaux l'ouverture d'un espace de cent places de vente autorisée, le Carré des biffins. La mobilisation tente ensuite de s'élargir à d'autres sites de marchés, notamment à Belleville. Elle y est relayée en 2011 par un collectif militant de soutien aux biffins créé à l'initiative d'élus locaux du Parti de Gauche (PG) ${ }^{2}$ et d'Europe Ecologie-Les Verts (EE-LV), partis de gauche défendant des alternatives aux positions du Parti socialiste et convergeant partiellement autour de valeurs anti-libérales, altermondialistes ou écologistes, voire autogestionnaires $^{3}$. Ce mouvement de défense d'un « droit à la ville » (Lefebvre, 1968 ; Harvey, 2011) pour ces vendeurs précaires met en avant leurs pratiques de récupération des déchets comme des résistances à la crise et à l'exclusion. Il défend la mise en place de " solutions locales pour un désordre global » (Serreau, 2010) comme alternative au gouvernement répressif des municipalités socialistes concernées (figure 1). Celles-ci appuient en effet les mouvements de défense du " cadre de vie » qui réclament la répression policière ${ }^{4}$, et dénoncent des nuisances (saleté, sentiment d'insécurité...) et une concurrence déloyale pour les commerçants, en articulant positions NIMBY ${ }^{5}$ et condamnation de cette économie de survie.

1 Il s'agit du « marché de l'Inquet » qui rassemble les dimanches matins, sur la place Arnaud Bernard, une cinquantaine de vendeurs d'articles d'occasion gérés par un organisme d'insertion et contrôlés par les placiers municipaux.

2 Créé le 1er février 2009, le Parti de Gauche, présidé par Jean-Luc Mélenchon (ex-PS) et Martine Billard (ex-Les Verts), est allié au Parti communiste (PC) et à d'autres organisations politiques de taille moindre au sein du Front de Gauche.

3 Depuis les années 1980, diverses coalitions politiques plus ou moins durables se sont revendiquées de la " gauche alternative ", rassemblant des partis et mouvements d'extrême-gauche de diverses tendances.

4 En vertu de la loi du 31 décembre 1982 dite loi « PLM » (Paris-Lyon-Marseille), les pouvoirs de police relèvent à Paris du ministère de l'Intérieur, et non des pouvoirs municipaux.

5 Acronyme de "Not In My Backyard" (" pas dans mon arrière-cour »), le phénomène NIMBY renvoie à une multiplicité de mobilisations locales contre des nuisances, allant de l'implantation d'équipements polluants à la présence de populations « indésirables ». 
Figure 1 : Les marchés informels à Paris : des mobilisations à la gestion parcellaire de l'entre-deux

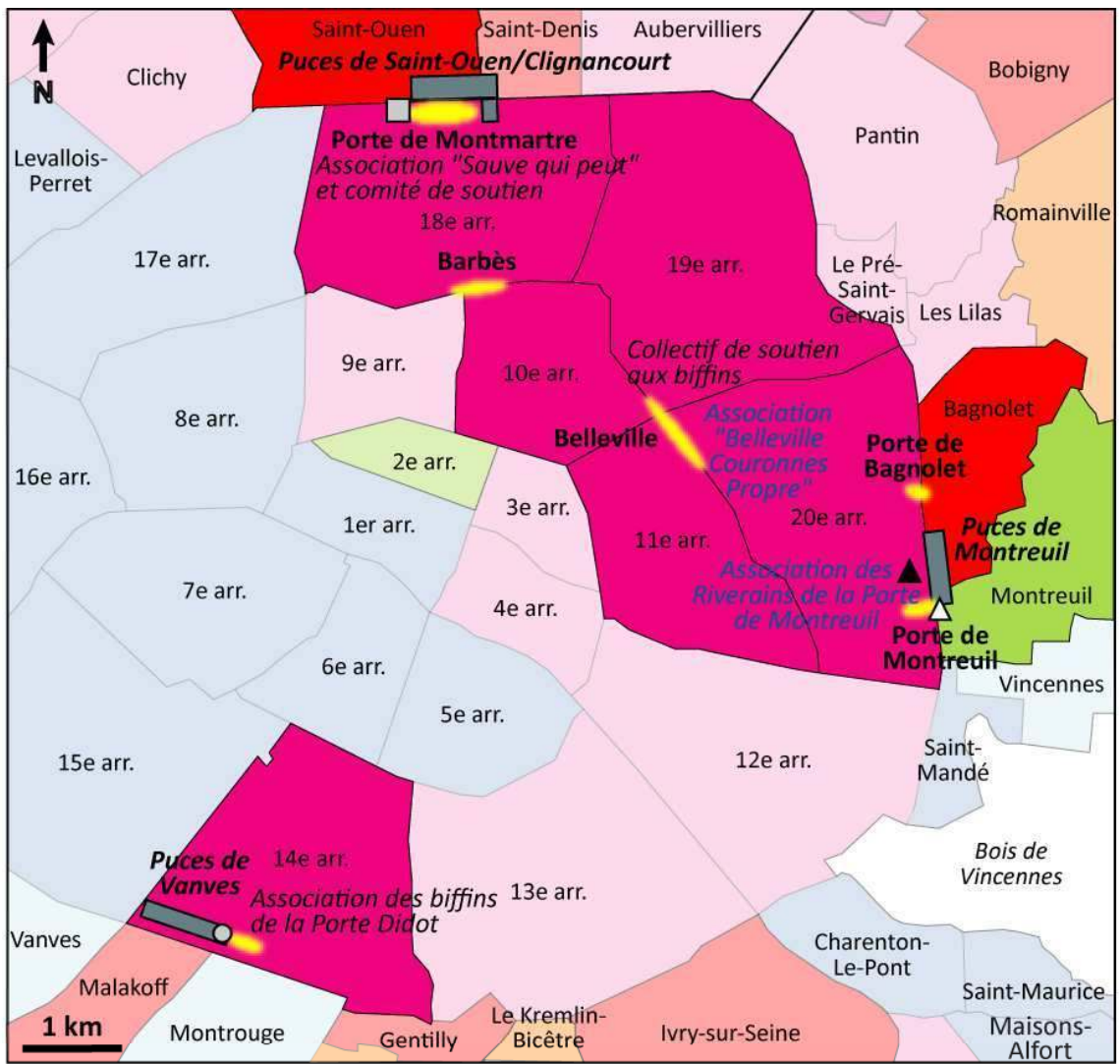

L'extension des marchés informels :

Marchés aux Puces

Présence continue ou intermittente d'un marché informel entre 2009 et 2012

Mobilisations et rivalités politico-territoriales autour des marchés informels :

Collectif Mobilisations des biffins de la Porte de Montmartre et de leurs relais

Association Mobilisations d'associations de défense du "cadre de vie"

Affiliation politique des maires des arrondissements parisiens et communes limitrophes au cours de la mandature 2008-2014:

Parti socialiste : - territoires concernés par la présence de marchés informels - territoires non concernés par la présence de marchés informels

Europe Ecologie - Les Verts : - territoires concernés par la présence de marchés informels - territoires non concernés par la présence de marchés informels

Parti communiste : - territoires concernés par la présence de marchés informels territoires non concernés par la présence de marchés informels

Autres partis de gauche

UMP

Autres partis de centre-droit

Une gestion locale par le biais d'intermédiaires :

Carré des biffins géré par l'association Aurore (100 places)

Puces de Vanves : espace réservé aux biffins (50 places)

$\Delta \Delta \quad$ Locaux de la "ressourcerie-recyclerie" gérée par l'association Emmaüs Coup de main : boutique / entrepot (en projet)

Carte : Balan H., 12/2013 
Ces marchés qui s'installent dans des espaces publics de quartiers parisiens péricentraux encore populaires sont des espaces de l'entre-deux à plusieurs niveaux. Ils marquent d'abord des interstices de la ville (Tonnelat, 2003), ceux du contrôle policier. Entre les épisodes de répression s'ouvrent des interstices temporels permettant l'installation des vendeurs sur des trottoirs ou des allées piétonnes en des lieux passants. La répression peut également provoquer le repli des vendeurs sur des lieux situés en retrait des voies principales. Ces interstices "s'opposent à l'espace qui détermine le caractère dominant de la ville (...) qui fixe la norme » (Hatzfeld, Hatzfeld, Ringart, 1998) : dans la ville-centre de la métropole, lieu de concentration de la richesse et du pouvoir, ces marchés "rendent de nouveau visible une précarité que l'on pensait marginale et régulée " (Milliot, 2010 : 18) dans les sociétés occidentales. Ils révèlent une économie de survie qui réapparaît dans le contexte des recompositions post-fordistes des économies métropolitaines (Sassen, 1998). Ces "paysages de la pauvreté " (Gervais-Lambony, 1994) évoquent les "problèmes communs d'insécurité et d'informalité " (Jacquot, 2007 : 181) associés aux villes du Sud comme archétype de la crise urbaine ${ }^{6}$, puisque " ces activités commerciales rendues à forme territoriale " s'inscrivent "hors des cadres spatiaux légaux, hors de l'empire des lois " (Péraldi, 2002 : 35). Les conflits qu'ils suscitent mettent en jeu non seulement les normes d'usage de l'espace public, mais aussi les normes sociales et socio-économiques associées au modèle urbain occidental. La maire socialiste du 20e arrondissement, Frédérique Calandra lance ainsi : "On est en France au 21e siècle, est-ce que, franchement, entretenir les chiffonniers du Caire ou l'équivalent, ça vous paraît un objectif social intéressant ? » (Milliot, 2011 : 46).

La défense d'une institutionnalisation de ces marchés informels à Paris ouvre alors une situation d'entre-deux gestionnaire et scalaire dans le gouvernement des espaces publics. L'informel, "notion opératoire » (Steck, 2003) au coeur de politiques publiques de développement visant un "secteur informel » massif au Sud, renvoie au Nord à des dynamiques traitées à la marge. C'est à l'occasion de conflits locaux que les responsables parisiens se trouvent confrontés à cette problématique ; l'affirmation politique des préoccupations locales est à l'arrière-plan des controverses autour de ces marchés. Pourtant, l'échelle métropolitaine constitue le cadre pratique des circuits d'approvisionnement et de déplacement des vendeurs et des chalands ${ }^{7}$, ainsi que le contexte socioéconomique de l'extension des marchés informels.

Les mouvements de récupérateurs qui émergent au Nord - biffins à Paris, binners ou " fouilleurs de poubelles » au Canada (Raoulx, 1999, 2006) - s'inscrivent dans un cadre politique de promotion d'un gouvernement urbain alternatif, fondé sur le local et la participation. Ils relaient les mobilisations défendant au Sud la reconnaissance des formes d'auto-organisation élaborées en réponse aux situations de crise, et leur inclusion dans une gestion urbaine plus participative. Dans le contexte parisien, l'investissement de ces espaces de l'entre-deux par des mouvements politiques qui les mettent en avant comme des alternatives locales à une crise métropolitaine et néolibérale donne-t-il

6 "Généralement les relations Nord-Sud sont abordées à l'échelle des Etats. La distinction Nord-Sud traduirait la domination économique et politique des pays du Nord sur ceux du Sud. Dans d'autres occurences, l'expression "du Sud" désigne une communauté de problèmes. Ainsi évoquer les "villes du Sud", par-delà les considérables différences entre Istanbul et Rio par exemple, c'est désigner d'un même mouvement les favelas et bidonvilles, les problèmes communs d'insécurité ou d'informalité. (...) A travers cette présentation apparaît une définition négative de ce que serait le Sud »(Jacquot, $2007:$ 181).

7 L'échelle métropolitaine correspond ici approximativement à la région Ile-de-France. 
lieu, face à la défense des normes du "cadre de vie ", à la construction d'un modèle alternatif de gouvernement urbain ? Comment le local, échelle des conflits autour de ces "territoires de l'informel » (Steck, 2003), est-il mis en avant comme échelle de l'alternative ? Quelles en sont les contradictions politiques ? Quels sont les nouveaux arrangements spatiaux qui en résultent ?

A la suite des travaux de Virginie Milliot ${ }^{8}$ sur la mobilisation des biffins, cet article s'appuie sur un travail de terrain réalisé en 2011-2012 . Celui-ci combine observation participante des activités du collectif de soutien aux biffins créé à Belleville en 2011, suivi des débats publics sur les marchés informels, entretiens auprès d'acteurs impliqués dans la controverse et échanges informels avec les protagonistes de la mobilisation des biffins. Après une présentation des conditions de l'institutionnalisation partielle des activités informelles des biffins, il s'agira de montrer comment un espace intermédiaire de mobilisation a pu se constituer malgré "l'interstitialité » de ces territoires marchands dont l'extension se confronte aux normes du «cadre de vie ». Enfin, les limites de la promotion de "solutions locales" seront mises en évidence par l'analyse des rivalités politicoterritoriales qui aboutissent à une gestion au cas par cas, locale et parcellaire.

\section{L'institutionnalisation partielle des pratiques informelles de vendeurs d'articles de récupération}

Parallèlement à l'extension des marchés informels parisiens, la pression exercée par la mobilisation des biffins amène les pouvoirs municipaux à mettre en place plusieurs dispositifs de gestion de leurs activités. Le nombre des vendeurs excède toutefois largement la taille de ces dispositifs, qui n'englobent pas la diversité des profils des populations présentes : la mobilisation des biffins n'obtient qu'une institutionnalisation partielle des pratiques informelles des vendeurs d'articles de récupération fréquentant ces marchés.

\section{Marchés informels parisiens et mobilisation des biffins}

En lle-de-France, la population fréquentant plus ou moins irrégulièrement les marchés informels est estimée à quelques milliers de personnes. En 2011, une fourchette de 1500 à 3000 vendeurs faisait consensus entre les participants du collectif de soutien aux biffins de Belleville en 2011, et la " cartographie des lieux de vente de récupérateurs-vendeurs sur le territoire francilien " commandée par la Région lle-de-France fait état d'une population observée de 2131 personnes (Rullac, Weiss, et al., $2012: 15)^{10}$. De plusieurs dizaines à plusieurs centaines de vendeurs s'installent en marge des marchés aux Puces pendant les trois jours d'ouverture de ceux-ci en fin de semaine, ainsi qu'à proximité des carrefours passants de Barbès et Belleville, en suivant le rythme bi-hebdomadaire des marchés alimentaires, et jusqu'à quotidiennement à Belleville en 2009-2011 (figure 1). La marchandise est déballée à même le sol sur des draps repliés en urgence lors des passages de police. Les fluctuations temporelles et spatiales de la présence des vendeurs suivent les aléas de la répression policière, à laquelle ces marchés informels résistent néanmoins. Les vendeurs se déplacent d'un site

8 Milliot, 2010, 2011, 2013

9 Les mandats municipaux mentionnés correspondent donc à la mandature 2008-2014.

10 Les auteurs font toutefois remarquer que " certains biffins exercent aléatoirement sur des sites différents qui sont proches, en fonction notamment des actions dissuasives de la police, des heurs et jours d'ouverture et de la présence des clients. Il est probable que la population réelle des biffins doit être minorée par rapport à notre comptage " (Rullac, Weiss, et al., $2012: 15)$. 
de marché à l'autre, ou investissent de nouveaux interstices spatio-temporels, se repliant sur des horaires nocturnes comme autour des Portes de Montreuil et de Bagnolet (figure 1).

Ces vendeurs présentent des profils très divers. Plusieurs études (ADIE, 2008 ; Rullac, Weiss, et al., 2012) soulignent néanmoins plusieurs points communs: la précarité et le rôle des difficultés financières dans le recours à la vente sur le marché informel, la surreprésentation des hommes retraités d'origine étrangère et l'importance des migrants en situation de grande précarité (dont l'origine change selon les mouvements migratoires internationaux). Cependant, plus que sur les profils sociologiques, la distinction s'opère entre anciens et nouveaux vendeurs. Cette opposition est souvent utilisée pour expliquer extension des marchés et tensions (par exemple avec les Roms récemment installés), mais elle est en partie une conséquence politique de la mobilisation des biffins, et de son traitement par les pouvoirs municipaux. L'ancienneté sur le marché et l'investissement dans la mobilisation des biffins ont servi à légitimer la reconnaissance d'une partie des vendeurs d'articles de récupération présents à la Porte de Montmartre, dits " historiques ». La mobilisation des biffins a été déclenchée en 2005 par l'intervention d'un élu écologiste du 18e arrondissement, lors d'une scène de répression policière du marché informel installé en marge des Puces de Saint-Ouen. Cet élu propose aux vendeurs d'articles d'occasion une réinterprétation de leurs activités : " ce que vous faites n'est pas mal, vous récupérez dans les poubelles » (Thierry Cayet in Milliot, 2010 : 44). En 2006, quelquesuns créent l'association de biffins Sauve qui peut. Le terme de biffin est un ancien nom des chiffonniers ayant perduré dans le jargon des Puces, avec une connotation parfois méprisante : "C'était un mot que je connaissais ; c'est dur de trouver des synonymes, mais "biffin", c'était le bouffon, quoi ", explique un enquêté. Repris et réinterprété par les écologistes locaux, le terme va au contraire " permett[re] de revaloriser l'activité de ces récupérateurs devenus invisibles derrière l'image du marché des voleurs " (Milliot, 2010 : 60). La promotion de la figure "verte " et vertueuse du biffin recycleur établit alors une distinction entre vendeurs d'articles de récupération et vendeurs de produits neufs, relayée par les acteurs militants puis reprise par les pouvoirs publics.

Sur ces marchés coexistent en effet des pratiques marchandes qui se différencient en partie par la nature et l'origine des produits vendus. Est ainsi distinguée la "sauvette ", vente d'articles de contrefaçon, de produits alimentaires et de consommation courante (sous-entendus " neufs »), de la " biffe ", " circuit de recyclage des objets" (Rullac, Bazin, et al., 2012). Les articles d'occasion sont largement issus de la fouille des poubelles, mais aussi de dons, de pratiques de rachat entre vendeurs, voire de la fouille des bennes de collecte de vêtements. Selon les comptages, prédomine soit la biffe (Rullac, Weiss, et al., 2012 ; collectif de soutien aux biffins de Belleville ; observations personnelles) soit la revente de produits neufs (association de défense du "cadre de vie " Belleville Couronnes Propre), ce qui traduit les différences de points de vue sur ces activités. Dans les faits, la distinction nette entre biffins et "vendeurs à la sauvette " gomme la complexité des modes de circulation et d'échange, et occulte l'existence de pratiques hybrides, y compris sur le Carré des biffins ${ }^{11}$.

11 Ainsi, pour leurs besoins personnels, des biffins s'approvisonnent sur ces marchés en produits alimentaires; " quelques vendeurs (...) ont trouvé des combines pour s'approvisionner dans des vestiaires pour SDF ou chez des grossistes " (Milliot, 2010 : 32), et " il est fréquent de voir sur le Carré des marchandises identiques à celles que l'on trouve sur le marché informel » (ibid. : 78). 


\section{Les dispositifs de gestion des biffins mis en place par les pouvoirs municipaux}

Sous la pression de la mobilisation des biffins, les pouvoirs municipaux parisiens ont mis en place trois dispositifs de gestion des biffins différents dans les $18 \mathrm{e}, 14 \mathrm{e}$ et $20 \mathrm{e}$ arrondissements. Ils donnent à voir des situations d'entre-deux gestionnaires, sans fournir de véritable alternative à l'occupation marchande informelle des espaces publics, notamment en raison de leur taille limitée (figure 2).

Un premier dispositif est mis en place à la fin de l'année 2009 dans le 18e arrondissement. Le Carré des biffins est un espace de cent places où la vente des articles de récupération est autorisée. Alors que plusieurs centaines de vendeurs sont présents à la Porte de Montmartre au moment de l'élaboration du Carré, les places sont réservées à des résidents des $18 \mathrm{e}$ et $17 \mathrm{e}$ arrondissements et de Saint-Ouen, retenus sur critères sociaux; les autres vendeurs en sont exclus ${ }^{12}$. La gestion de ce " dispositif d'expérimentation sociale » mis en place par les pouvoirs municipaux est confiée, sur appel d'offres, à une association d'action sociale. L'association Aurore, structure employant plus de 1000 salariés et assurant des délégations de service public dans les domaines de l'hébergement, de la santé et de l'insertion, est chargée de l'attribution des places, du respect de la charte réglementant le Carré ainsi que d'une mission d'accès aux droits et d'insertion auprès des biffins. Créé par décret municipal, le Carré des biffins a le statut dérogatoire de service de l'association Aurore les jours d'ouverture, suivant ceux des Puces de Saint-Ouen. II est réglementé par une charte à laquelle les biffins adhèrent, sans être adhérents de l'association Aurore. Cette charte proscrit la vente de produits neufs, alimentaires et cosmétiques, suivant "des critères convenus avec la municipalité qui ne s'inscrivent absolument pas dans le registre du commerce ", selon les mots de la salariée d'Aurore responsable du Carré. Ce premier dispositif consiste donc en un encadrement, dans l'espace public, des activités informelles d'un nombre limité de biffins, justifié au nom de l'action sociale.

Un second dispositif consistant en un simple encadrement des activités informelles d'un nombre limité de biffins est mis en place en 2012 aux Puces de Vanves. Toutefois, à la différence du Carré des biffins, installé dans l'espace public, ce dispositif tente de contenir l'extension de la vente informelle en l'insérant dans les Puces. Les revendications de l'Association des biffins de la Porte Didot ${ }^{13}$ ont abouti à une redéfinition partielle de la gestion interne des Puces de Vanves, déléguée à une entreprise gestionnaire (la SEMACO) : cinquante places sont réservées, l'après-midi, à des biffins, pour un coût de deux euros. Mais ce dispositif provoque des conflits avec les brocanteurs et commerçants patentés autour des règles d'attribution des places, et celles-ci restent parfois inoccupées tandis que les vendeurs informels continuent à s'installer en marge des Puces.

Enfin, dans le 20e arrondissement, la municipalité a ouvert en 2012-2013 une "ressourcerierecyclerie ", lieu de retape et revente d'articles et de matériaux de récupération, s'inscrivant dans le cadre du plan régional d'élimination des déchets en lle-de-France de 2009 qui prévoit l'ouverture de 30 ressourceries à l'horizon 2019. La ressourcerie est censée fournir une trentaine d'emplois d'insertion à des biffins mais aussi à des habitants du quartier ${ }^{14}$, et encadrer les activités de

12 La présence des biffins " titulaires " d'une place est toutefois irrégulière, et l'association Aurore redistribue à l'ouverture du "Carré" les places laissées vacantes à des vendeurs adhérant à la charte du Carré des biffins et autorisés à s'y installer à titre journalier.

13 Cette association représente des biffins présents en marge des Puces de Vanves. Elle n'a pas de liens établis avec l'association de biffins Sauve qui peut de la Porte de Montmartre.

14 A l'ouverture de la recyclerie en mars 2014, celle-ci offre 10 emplois d'insertion, devant être portés à 30 en juin 2014. 
récupération des biffins par un circuit court : "Nous allons créer un lieu de tri ou des gens pourront se faire payer (...) de la matière première qu'ils auront glanée ", annonce la maire du 20e arrondissement fin 2011. La gestion en est déléguée, sur appel d'offres, à l'association Emmaüs Coup de main, employant quelques dizaines de salariés et membre du réseau Emmaüs depuis 2010, qui menait à l'origine des actions d'insertion auprès des Roms roumains en Seine-Saint-Denis. Ce projet de circuit court, centré sur les pratiques de récupération des biffins et non plus sur leurs pratiques marchandes, reste aujourd'hui encore en suspens. S'il vise, à l'instar du dispositif des Puces de Vanves, à limiter l'occupation de l'espace public, il s'inscrit dans la perspective d'une gestion alternative, locale et artisanale des déchets. L'installation d'un local de la ressourcerie à la Porte de Montreuil, sur un territoire de vente informelle en marge des Puces, peut néanmoins apparaître comme un outil de contrôle territorial plutôt que d'intégration des biffins.

La mobilisation des biffins aboutit donc à l'élaboration de dispositifs gestionnaires parcellaires dans les principaux arrondissements concernés par la présence des marchés informels (figure 2). Les pressions exercées par les acteurs investis dans cette mobilisation se confrontent, notamment dans le 20e arrondissement, à des mouvements de défense du "cadre de vie " qui limitent leur portée ; ceux-ci réclament au contraire la répression policière ces marchés.

Figure 2 : Les marchés informels parisiens entre mobilisations et institutionnalisation

\begin{tabular}{|c|c|c|c|c|c|}
\hline $\begin{array}{l}\text { Site de marché } \\
\text { informel }\end{array}$ & $\begin{array}{l}\text { Arrts. } \\
\text { concernés }\end{array}$ & $\begin{array}{l}\text { Mouvement de } \\
\text { soutien aux biffins }\end{array}$ & $\begin{array}{c}\text { Mouvement } \\
\text { organisé } \\
\text { de défense } \\
\text { du « cadre de vie » }\end{array}$ & $\begin{array}{l}\text { Dispositif de } \\
\text { gestion des biffins }\end{array}$ & $\begin{array}{c}\text { Acteur } \\
\text { intermédiaire } \\
\text { délégataire }\end{array}$ \\
\hline $\begin{array}{c}\text { Porte de } \\
\text { Montmartre }\end{array}$ & $18 \mathrm{e}$ & $\begin{array}{l}\text { Association de } \\
\text { biffins « Sauve qui } \\
\text { peut »; comité de } \\
\text { soutien aux biffins }\end{array}$ & - & «Carré des biffins » & $\begin{array}{l}\text { Association } \\
\text { «Aurore » }\end{array}$ \\
\hline $\begin{array}{c}\text { Portes } \\
\text { de Montreuil } \\
\text { et de Bagnolet }\end{array}$ & $20 e$ & - & $\begin{array}{l}\text { "Association des } \\
\text { Riverains de la } \\
\text { Porte de } \\
\text { Montmartre" }\end{array}$ & $\begin{array}{l}\text { Ressourcerie : } \\
\text { emplois d'insertion } \\
\text { et projet de "circuit } \\
\text { court" de recyclage }\end{array}$ & $\begin{array}{c}\text { Association } \\
\text { « Emmaüs Coup de } \\
\text { main » }\end{array}$ \\
\hline Belleville & $\begin{array}{c}20 e, 11 e \\
10 e, 19 e\end{array}$ & $\begin{array}{l}\text { Collectif de soutien } \\
\text { aux biffins }\end{array}$ & $\begin{array}{c}\text { Association } \\
\text { « Belleville } \\
\text { Couronnes Propre » }\end{array}$ & - & - \\
\hline $\begin{array}{l}\text { Porte de } \\
\text { Vanves }\end{array}$ & $14 \mathrm{e}$ & $\begin{array}{c}\text { "Association des } \\
\text { biffins de la Porte } \\
\text { Didot " }\end{array}$ & - & $\begin{array}{l}\text { Attribution de } 50 \\
\text { places au sein des } \\
\text { Puces de Vanves }\end{array}$ & $\begin{array}{l}\text { SEMACO (Société } \\
\text { d'exploitation de } \\
\text { marchés } \\
\text { communaux) }\end{array}$ \\
\hline
\end{tabular}

Les marchés informels parisiens entre intermédiaires et interstices : la défense locale d'une alternative face aux conflits autour de l'espace public

Les controverses autour de l'occupation des espaces publics par les marchés informels opposent donc deux tendances contraires : la défense des biffins et la défense du "cadre de vie ". Alors que le 
redéveloppement des activités informelles sous forme de marchés se confronte aux normes dominantes qui règlent les usages des espaces publics, la brutalité policière à l'encontre de personnes visiblement démunies peut aussi déclencher une " solidarité réactive » (Milliot, $2010: 22$ ), à l'origine de la mobilisation des biffins.

\section{La constitution d'un espace intermédiaire de mobilisation à la Porte de Montmartre}

La mobilisation des biffins s'est ainsi formée en réaction à la brutalité croissante de la répression policière envers les vendeurs informels, et a ouvert un espace intermédiaire de négociation politique. La vente sur le marché informel est mise en avant comme une alternative pratique à la crise ; ainsi, explique une élue écologiste,

"le Parti socialiste voit les biffins comme une résurgence du passé, alors que moi, je les vois plutôt comme des inventeurs d'avenir, comme des gens qui sont en train de créer des activités dont on va avoir besoin dans pas très longtemps, parce qu'on va manquer de ressources, parce qu'on va être obligé de retrouver des ressources sur le territoire (...). Pour moi, les biffins, c'est le futur ».

La valorisation de la dignité de ces vendeurs précaires fait écho aux mouvements sociaux sudaméricains, fondés "sur le rejet de l'exclusion et la notion de dignité " (Canovas, 2008 : 43), dans un contexte où "la population pauvre et marginalisée, dénuée de ressource, a dû s'organiser pour survivre ", et où "l'indifférence des pouvoirs publics et de la classe aisée a laissé place à une guerre à I'[...] encontre [de cette population] afin d'estomper, voire de cacher une réalité sociale dramatique " (ibid. : 45). La mobilisation des biffins s'appuie sur la diffusion des revendications en termes de " droit à la ville " pour ouvrir dans le 18e arrondissement un espace intermédiaire de négociation des normes. Celui-ci permet de composer un " arrangement local » (Milliot, 2010 : 56) avec le modèle urbain dominant : les Verts défendent "l'idée d'une solution locale, d'un arrangement pragmatique permettant de régler ce problème "à échelle humaine" sous la forme d'un marché social de la récupération » (ibid.).

La mobilisation des biffins de la Porte de Montmartre joue à la fois sur l'échelle micro-locale du quartier, par la médiation auprès des riverains, et sur les ressorts supra-locaux que constituent les débats politiques au Conseil de Paris et les relais médiatiques. Ce sont d'abord les facteurs locaux qui ont permis la mobilisation : la défense d'une reconnaissance locale des biffins a trouvé un écho dans la culture socio-politique locale, celle d'un quartier en position de marge péricentrale, où la médiation par les instances de la vie de quartier l'emporte sur le recours aux pouvoirs municipaux. Les "querelles idéologiques" (ibid. : 50) et les réticences de la municipalité du 18e arrondissement à institutionnaliser l'informel y ont été contournées par la défense d'un droit coutumier local, les biffins apparaissant comme les descendants des chiffonniers présents à la création des Puces de Saint-Ouen. Ce " retournement du stigmate » (Milliot, $2010: 60$ ) a été possible parce que le travail de médiation effectué autour de la figure du biffin a réinscrit celle-ci dans la culture de ce quartier où les petites activités de rue ont été rejetées avec la mise en place des marchés aux Puces :

"Ce terme a permis de construire une continuité historique, une identité d'expériences avec les personnes âgées du quartier qui avaient toutes connu l'univers de la biffe sur lequel se sont érigées les Puces. Il ouvrait un espace ou chacun pouvait se reconnaître des valeurs partagées de débrouillardise, d'autonomie par le travail, de lutte contre le gaspillage, etc » (ibid.).

L'association de biffins Sauve qui peut sera ainsi soutenue par un collectif composé de militants politiques et associatifs, mais aussi de riverains, d'amicales de locataires et d'habitués du marché. La mobilisation est relayée et appuyée par le conseil de quartier, l'équipe de développement local dédiée 
à ce quartier classé en Politique de la Ville, le café associatif Le Petit Ney... Néanmoins, en 2008, il s'en faut de peu que la soudaine extension du marché informel jusqu'au mail Binet - " poumon vert du quartier ", selon le président du Petit Ney - ne mette à mal ce travail de médiation. Des riverains lancent en effet un mouvement de défense de leur " cadre de vie " en installant sur le mail des tables et des chaises pour délimiter leur territoire face à l'installation des vendeurs. Mais les biffins restent en position de force sur le plan politique, puisque cette occupation du terrain se fait sans recours à la municipalité du 18e arrondissement : les riverains manifestent sur le terrain, les biffins devant la mairie. La mobilisation de ces derniers finit par aboutir, dans le contexte des élections municipales de 2008, à la mise en place d'un espace de vente autorisée, le Carré des biffins. Toutefois, la vulnérabilité du local face aux enjeux supra-locaux des marchés informels, que le conflit autour du mail Binet laissait présager, refait surface avec la mise en place du Carré, dont la taille et les critères d'accès excluent la majorité des vendeurs. Peu avant son ouverture, les militants de la Porte de Montmartre tentent de mobiliser sur d'autres sites de marché, sans grand succès :

"Ils ont essayé de me motiver pour que je ramène des gens de Belleville sur Porte Montmartre, en échange de quoi eux, de Porte Montmartre, viendraient à Belleville, un truc de ce goût-là, solidarité, machin... Alors je suis allé à une ou deux manifestations riquiqui tambouriner, mais peu de gens de Belleville se déplaçaient là-bas ", se souvient Nicolas.

Construite dans un rapport d'opposition des Verts au Parti socialiste au sein de la majorité municipale du 18e arrondissement, la mobilisation des biffins impute la responsabilité du désordre provoqué par le marché informel aux pouvoirs municipaux et à leur politique répressive, et prône une solution locale. En prenant à la lettre ce discours, la municipalité socialiste du $18 \mathrm{e}$ arrondissement met en place une gestion qui provoque la division de la mobilisation ; le Carré des biffins est ainsi qualifié par certains militants du comité de soutien aux biffins de " piège de la municipalité » (Milliot, $2010: 68$ ). Ce « dispositif d'expérimentation sociale » reprend dans sa formulation l'idéologie développée par le Parti socialiste dans les années 1970 autour du local, représentant " potentiellement, une expérience porteuse de nouveaux rapports sociaux, de recherche de nouvelles formes de vie sociale et un laboratoire d'expérimentation sociale » (Lefebvre, 2011 : 70). Mais dans sa mise en pratique, le Carré apparaît plutôt comme une tentative de cantonnement de ces "territoires de l'informel " (Steck, 2003), qui révèle les contradictions de la promotion du local comme échelle privilégiée de l'alternative. Alors que la répression s'est renforcée autour du Carré " qui n'a cessé de déborder » (Milliot, 2010 : 81), le maire du 18e arrondissement, Daniel Vaillant incite régulièrement la mobilisation des biffins à réclamer ailleurs l'ouverture de nouveaux espaces de vente, et insiste sur les limites de ses compétences: "Nous ne prétendons pas régler, avec les prérogatives qui sont les nôtres, les problèmes de la misère, les problèmes de l'exclusion, le problème de l'utilisation de l'espace public, voire de l'ordre public ", déclarait-il en 2011 lors du bilan de fonctionnement du Carré, deux ans après son ouverture.

Ces usages informels des espaces publics perturbent en effet la norme de la ville occidentale.

\section{Des espaces interstitiels confrontés aux normes du " cadre de vie »}

Dans la métropole européenne qui fut au 19e siècle la "capitale de la modernité » (Harvey, 2012), l'extension récente de ces marchés (figure 3 ) va à rebours de la réduction des tolérances envers les usages marchands informels des espaces publics. Ces espaces publics sont définis par la modernité occidentale comme des espaces "vides » dédiés aux fonctions de circulation (Choay, 1994 ; Vallat et 
al., 2008). La confrontation entre cette évolution normative à long terme et le retour de pratiques de survie urbaine déclenche des conflits territoriaux locaux. Dans le contexte du " déclin des illusions étatistes " (Oblet, $2005: 168)^{15}$, puis du bouleversement des rapports de production qui organisaient les sociétés fordistes, le local a été promu comme "cadre de vie " par les luttes urbaines des classes moyennes (ibid.), qui montent en puissance depuis les années 1970. Les valeurs attachées à " l'image de la ville " (Lynch, 1960) sont alors perturbées par le retour dans les espaces péricentraux des petites activités de rue qui avaient été rejetées aux marges physiques et normatives de la ville occidentale.

Ces petites activités de rue ont en effet été repoussées du centre de Paris à partir du 19e siècle (Charpy, 2011). Devenues hors normes, elles sont cantonnées à la fin du 19e siècle dans les marchés aux Puces fixés à certaines portes de la ville. Là, des zones de vente informelle « intégrées à la marge au fonctionnement des Puces " (Milliot, 2010 : 10) se maintiennent jusqu'à nos jours en bordure des Puces, bien que celles-ci aient évolué au 20e siècle vers la vente d'articles de brocante et plus récemment de produits neufs, souvent sous la bannière discount. La vente de rue persiste en outre assez longtemps de manière diffuse dans certains quartiers populaires. Sur le boulevard de Belleville (figure 3), raconte Nicolas, biffin habitant le quartier,

"sur ces trente dernières années, il y a toujours eu des gens qui vendaient à la faveur des bancs publics (...). Tu te promenais sur le boulevard, et il y avait des gens qui vendaient de tout. "

\section{Figure 3 : Marché informel à Belleville, 19e arrondissement}

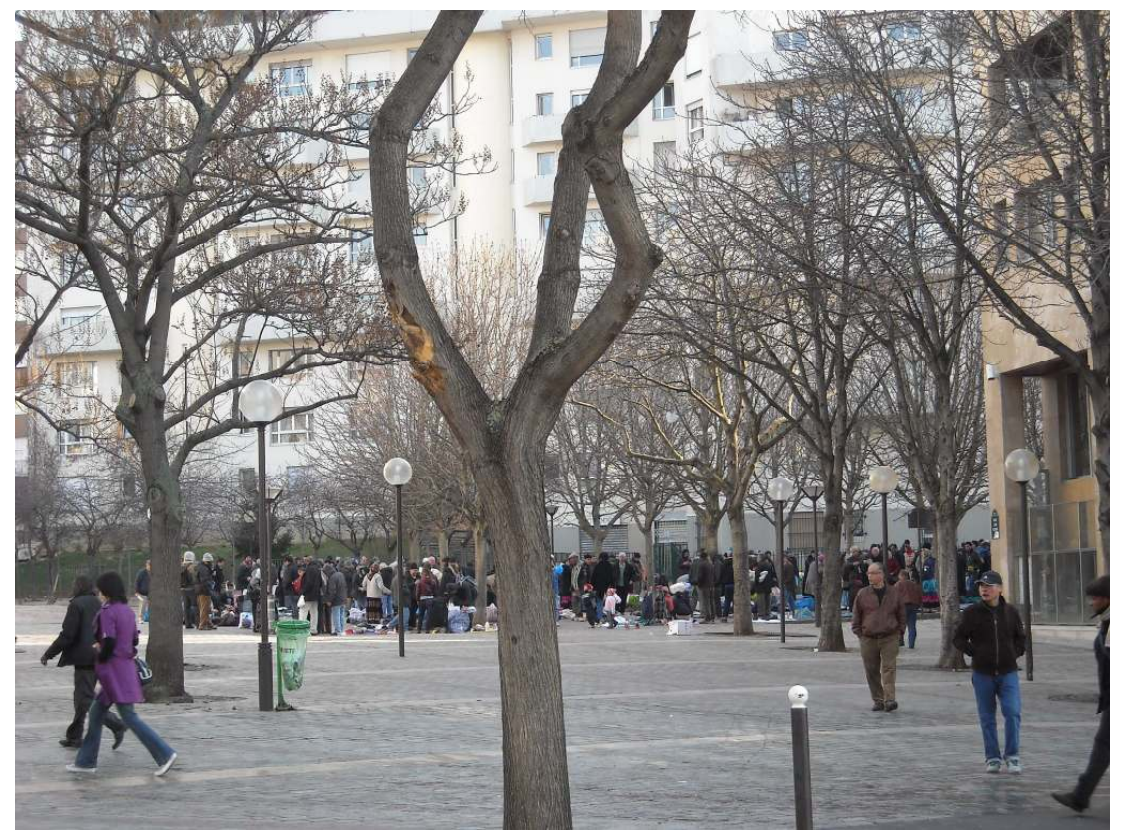

Cliché : Balan H., 16/03/2010

Mais ces activités diffuses sont progressivement compressées par le durcissement des politiques sécuritaires, qui accompagne l'embellissement et la normalisation des anciens quartiers populaires de Paris (Clerval, 2013), et qui entraîne la multiplication des opérations visant à limiter les usages

15 « A partir de 1975, avec le déclin des illusions étatistes, les luttes urbaines changent de nature. (...) Les mouvements sociaux de lutte pour le "cadre de vie" se multiplient. (...) Quelle que soit la diversité de ces mouvements, une composante militante s'en détache : elle est issue des classes moyennes » (Oblet, 2005 : 168-169). 
"indésirables ${ }^{16}$ des espaces publics - comme le retrait des bancs publics. Le processus se heurte toutefois, à partir des années 1970, à un mouvement contraire de redéveloppement des petites activités de rue, qui investissent les espaces souterrains du métro (Coste, 1994), les sites touristiques, ainsi que les quartiers de commerce dit " ethnique ", où elles suscitent des conflits autour des usages légitimes de l'espace public ${ }^{17}$. Dans les anciens quartiers de vente diffuse, cette pression semble avoir provoqué le rassemblement des vendeurs, formant de petits marchés qui se sont étendus en 2008, avec la crise économique mais peut-être aussi avec la médiatisation de la mobilisation des biffins, qui laissait présager une "détente ". L'occupation du boulevard par le marché informel rend soudainement visibles des pratiques marchandes qui passaient relativement inaperçues auparavant. Elles deviennent l'objet de controverses publiques en se confrontant aux normes du "cadre de vie "; le maire du 11e arrondissement, Patrick Bloche, déclare :

"Je connais le boulevard de Belleville depuis 33 ans. II y a toujours eu dans l'après-midi, tranquillement, quelques petits vendeurs, souvent des vieux migrants, qui faisaient du troc, des petites ventes, etc. Tant que c'était une occupation de l'espace public régulée, acceptable, aucun riverain, aucun commerçant ne s'est levé en disant, c'est inacceptable, c'est insupportable! C'est parce que c'est devenu un mouvement de masse que la vie est devenue insupportable pour les habitants, et qu'il y a eu interpellation des pouvoirs publics. "

L'occupation de trottoirs ou d'allées piétonnes par les marchés informels entrave la fonction de circulation des espaces publics et bouleverse le paysage urbain. Elle déclenche les protestations de riverains qui demandent le rétablissement de conditions de circulation et de propreté " normales ", et l'aménagement des espaces publics concernés. Ces revendications en matière de "cadre de vie " local sont appuyées par un discours sur l'équité territoriale, qui prend comme norme de référence l'image valorisée des "beaux quartiers" (Raoulx, 2006). Un riverain de la Porte de Montmartre demande ainsi, lors d'un conseil de quartier en 2011,

"l'aménagement du mail Binet ${ }^{18}$, parce que nous avons le droit à des rotondes, à des fleurs, à des bancs", arguant que "dans les HBM qui bordent tout le boulevard Ney, il y a beaucoup de précarité sociale, il y a des gens qui ne peuvent pas s'aérer en partant sur les Champs-Elysées ou en se permettant de faire des sorties à l'extérieur du 18e, et qui aimeraient simplement sortir avec leur famille, avec leurs enfants, sur le mail Binet, histoire de s'aérer quand il fait soleil ».

L'extension des marchés informels submerge les dernières tolérances locales, provoquant des réactions de rejet et un sentiment d'insécurité associés à une "peur du déclassement " (Milliot, 2010 : 17). Dans le quartier de logements sociaux de la Porte de Montmartre, celle-ci correspond à une "peur de basculer de peu à rien» (ibid.) ; dans le quartier de Belleville, gagné par l'embourgeoisement du nord-est parisien, elle touche aux valeurs de l'immobilier.

L'extension des marchés informels est interprétée comme un signe de disqualification socio-politique dans ces anciens quartiers populaires péricentraux, où la valeur sociale de "l'image de la ville» (Lynch, 1960) s'est affirmée parallèlement à l'arrivée des classes moyennes auxquelles le Parti socialiste s'est allié par la valorisation politique du «cadre de vie » local (Lefebvre, 2011). Cette

16 Voir le travail de thèse (en cours) de Muriel Froment-Meurice sur « la gestion des usages indésirables dans les espaces publics parisiens $"$.

17 C'est notamment le cas dans les quartiers de la Goutte d'Or et de Château-Rouge, dans le 18e arrondissement (Bacqué, 2006 ; Chabrol, 2012).

18 Le mail Binet est une large rue occupée sur la moitié par une allée plantée, sur laquelle s'est étendu le marché informel de la Porte de Montmartre. 
valorisation socio-politique s'est partiellement diffusée aux habitants moins aisés ${ }^{19}$. La politique des maires socialistes consiste aujourd'hui à relayer les revendications des riverains pour mettre en place des actions de répression des marchés informels, réagissant de manière défensive à la vulnérabilité socio-économique que ces marchés révèlent tant chez les vendeurs que chez les chalands. Transposant les revendications NIMBY en politique municipale, la maire socialiste du 20e arrondissement Frédérique Calandra déclarait en 2011 que "le 20e ne doit pas être le paillasson sur lequel le préfet de Paris s'essuie les pieds ". Les marchés informels sont présentés comme la conséquence d'un laxisme de la préfecture de Paris visant à dégrader les anciens quartiers populaires du nord-est de Paris ; ils deviennent, au nom de l'équité territoriale, des stigmates territoriaux, dans une rivalité avec les traditionnels territoires «bourgeois » de la droite parisienne. Ceci illustre, selon Danielle Simonnet, élue du Parti de $\mathrm{Gauche}^{20}$, "la dérive sécuritaire que peut avoir la socialdémocratie », et dont Frédérique Calandra incarnerait « la phase avancée sur Paris ».

Les limites à la poursuite de cette mobilisation dans d'autres contextes locaux, où la conjoncture socio-politique est nettement défavorable à la reconnaissance des biffins, montrent alors les contradictions d'une institutionnalisation envisagée par le prisme politique du local.

\section{Démocratie locale et gestion urbaine des marchés de biffins : entre-deux scalaire et jeux d'acteurs}

La mobilisation de la Porte de Montmartre a amorcé un travail local de négociation des normes dominantes qui règlent les usages des espaces publics. Toutefois, après la mise en place du Carré des biffins, elle se trouve confrontée à une complexification des enjeux scalaires et des jeux d'acteurs. L'embarras des responsables municipaux face à la vulnérabilité socio-économique que ces marchés rendent visible aboutit à une gestion locale, au cas par cas et à la marge, par le biais d'intermédiaires. Les rivalités locales apparaissent en effet peu propices à l'élaboration d'une alternative prenant en compte les enjeux supra-locaux de l'extension des marchés informels parisiens.

\section{Les suites de la mobilisation des biffins : l'alternative confrontée aux rivalités politico-territoriales locales}

La défense des biffins connaît une montée en échelle, qui se heurte néanmoins, au-delà du contexte de la Porte de Montmartre, à des alliances locales d'opposition à la création de nouveaux espaces de vente. A partir de 2009, l'association Sauve qui peut participe aux rencontres franco-brésiliennes "Déchets et citoyenneté " organisées par la fondation France Libertés ${ }^{21}$ avec des réseaux internationaux de récupérateurs et de vendeurs de rue. La question des biffins s'inscrit donc par ce biais dans des réflexions plus larges sur les possibilités de réorganisation de l'économie sociale et

19 Dans les années 1990, l'association La Bellevilleuse, qui s'oppose au projet de rénovation urbaine de Belleville, utilise ainsi l'amélioration de la propreté du quartier comme un outil d'empowerment des riverains des classes populaires (Fayman, 1990)

20 Et candidate du Parti de Gauche à la mairie de Paris pour les élections municipales de 2014.

21 La fondation France Libertés a été créée en 1986 par Danielle Mitterrand. Le programme « Déchets et citoyenneté » de la fondation est créé suite à un travail mené par la fondation avec les catadores brésiliens, ramasseurs informels dans les déchetteries organisés en coopératives, dans l'idée que "I'activité des biffins et des Roms ici en France nous rappelle que la valorisation des déchets ne peut être réduite à une approche technique et industrielle " (http://www.francelibertes.org/-Dechets-et-Citoyennete,33-.html\#). 
solidaire des déchets. Parallèlement, la mobilisation des élus EE-LV s'affirme dans les instances supralocales du Conseil de Paris puis du Conseil régional d'lle-de-France. Sous leur pression, ce dernier vote pour l'année 2011 un budget d'un million d'euros dédié à la mise en place d'alternatives à la répression des biffins.

Mais l'élaboration d'alternatives reste tributaire des pouvoirs municipaux qui ont compétence sur l'espace public. Or, dans le 20e arrondissement, la mobilisation des biffins est confrontée à l'alliance des associations de défense du " cadre de vie » avec la maire socialiste Frédérique Calandra. Celle-ci organise en 2010 et 2011, avec les commerçants des Puces de Montreuil, I'Amicale des Riverains de la Porte de Montreuil et l'association Belleville Couronnes Propre, des manifestations visant à obtenir de la Préfecture de police une répression constante des marchés informels dans le 20e arrondissement. La mise en avant du local avait permis de surmonter, dans le 18e arrondissement, les réticences de la municipalité à institutionnaliser l'informel ; mais elle devient ailleurs un outil d'opposition à la reconnaissance des biffins.

La mise en place de dispositifs gestionnaires institutionnalisant partiellement les pratiques informelles des biffins suppose en effet une distinction entre biffins et "vendeurs à la sauvette ", sous-entendu d'articles neufs; celle-ci permet alors la diabolisation de ces derniers par contraste avec la figure vertueuse du biffin recycleur et sert à justifier, par extension, le rejet de tous les vendeurs informels. La répartition des articles proposés sur ces marchés entre récupération et neuf est donc devenue, par la mobilisation et l'institutionnalisation, un enjeu politique. L'Association Bellevilles Couronnes Propre, pour avancer que les vendeurs présents sur le marché informel de Belleville ne sont pas des biffins et pour s'opposer à l'ouverture d'un espace de vente, s'appuie non seulement sur les comptages qu'elle réalise ${ }^{22}$, mais aussi sur les propos du maire du $18 \mathrm{e}$ arrondissement. Celui-ci répète que le Carré des biffins est destiné à " un public bien spécifique et particulier dans le $18 \mathrm{e}$ arrondissement ", étayant le refus que le dispositif soit reproduit sur les autres sites de marchés informels.

\section{Figures 4 et 5 : Manifestation de l'association Belleville Couronnes Propre sur le boulevard de Belleville, en présence des maires des $20 \mathrm{e}, 11 \mathrm{e}$ et $10 \mathrm{e}$ arrondissements}
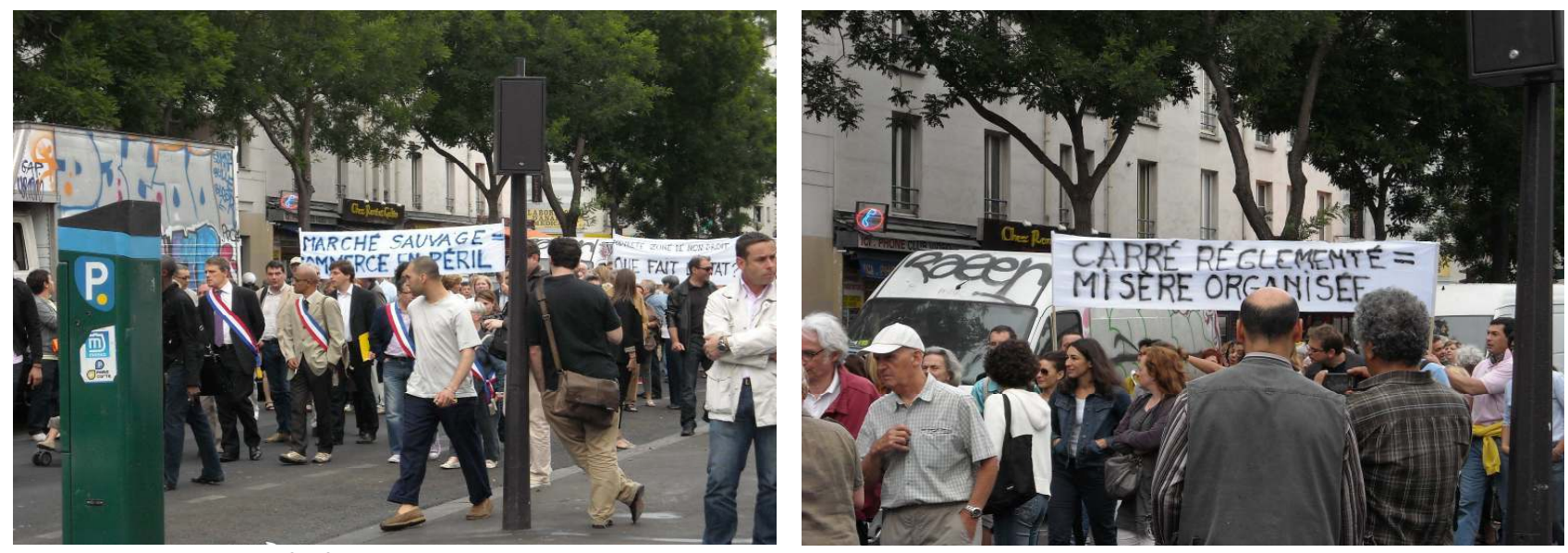

Clichés : Balan H., 20/05/2011

$22 \mathrm{Cf}$ supra. Ces comptages, démentis par le collectif de soutien aux biffins, tendent à montrer que les vendeurs d'articles de récupération sont en minorité sur le marché informel du boulevard de Belleville. 
En réaction aux prises de position répressives de la maire du 20e arrondissement, les élus EE-LV et PG, composantes minoritaires de la majorité municipale, multiplient les pressions pour l'ouverture de marchés de biffins au Conseil de Paris, au Conseil régional d'lle-de-France, et auprès des autres maires concernés. En 2011, ils s'allient aux militants de la Porte de Montmartre pour créer à Belleville un collectif de soutien aux biffins "sur une opposition de gauche à la majorité de gauche ", selon un participant.

Les discours sur le local que mobilisent ces partis de gauche reprennent des éléments développés par le PS dans les années 1970 . Les élus EE-LV réinterprètent le thème de l'autogestion communale, utilisé auparavant par le PS « pour se démarquer du vocabulaire de la droite qui a investi le thème de la participation » (Lefebvre, 2011 : 70), en critiquant le Parti socialiste pour sa " façon de toujours vouloir traiter les problèmes de façon industrielle, ou généraliste ", selon les mots d'une élue du 20e arrondissement. Le PG reprend d'autres éléments ; dans le discours de Danielle Simonnet, conseillèreadjointe PG du 20e arrondissement et conseillère de Paris, "le "local" est à la fois conçu comme un lieu politique en soi (dans le sens où peut s'y produire une cohérence globale) et n'ayant de signification que dans une perspective politique plus large " (Lefebvre, 2011 : 69). Elle expose la possibilité d'un changement global qui rendrait la marge gérable :

"On doit pouvoir trouver des minima sociaux qui permettent de vivre, ce qui ne veut pas dire qu'on arrêtera la biffe ; mais, du coup, elle change de nature, elle redevient un petit marché de la récup', de petite taille, en complément, et une activité sociale qui a aussi une vertu écologique - autre chose qu'une survie pour les vendeurs et une consommation alternative de survie pour les acheteurs".

Or, les rivalités territoriales qui se jouent autour de l'extension des marchés informels entre arrondissements parisiens socialistes, et communes limitrophes gouvernées par des maires EE-LV et PC (figure 1), révèlent les limites des discours sur le local. Comme au PS dans les années 1970, ceux-ci fonctionnent "comme un discours de subversion des équilibres politiques établis, de contestation des "notables" en place, bref comme un discours d'accès au jeu politique » (Lefebvre, 2011 : 79). Les rivalités autour de l'accès aux responsabilités municipales limitent alors l'élaboration d'alternatives locales. La peur que les "nuisances " des marchés informels n'entraînent une marginalisation des territoires concernés met en défaut la promotion du local comme échelle privilégiée de l'alternative portée par les écologistes, révélant l'importance électorale des préoccupations en matière de " cadre de vie ". Ainsi, Dominique Voynet, maire EE-LV de Montreuil, refusait de créer un marché pour les biffins sans coordination avec les municipalités voisines - et hostiles à la gestion des activités marchandes des biffins - de Bagnolet (PC) et du 20e arrondissement (PS) ; elle justifiait son refus par la crainte que les activités informelles ne soient rejetées à la périphérie tandis que le 20e arrondissement proposerait "des manifestations artistiques, des expositions d'antiquaires » plus prestigieuses.

Les controverses autour de la question des biffins s'inscrivent donc dans une contradiction entre la promotion politique de l'échelle locale comme échelle privilégiée de l'alternative, et les rivalités territoriales en jeu à cette échelle. Les enjeux politico-territoriaux locaux favorisent l'affirmation de la gestion au cas par cas comme priorité politique, au détriment de la constitution d'une réelle alternative. 


\section{Gestion au cas par cas et interposition d'intermédiaires : le local, limite à l'alternative ?}

Les pressions exercées en faveur des biffins aboutissent à une gestion au cas par cas qui varie selon les contextes politiques micro-locaux. Le contournement des "querelles idéologiques » (Milliot, 2011 : 62) sur l'informel par la mise en place d'un « arrangement local » (ibid. : 57) trouve ses limites dans la démultiplication de dispositifs parcellaires. En outre, l'appréhension de la gestion urbaine des marchés informels par le prisme du local apparaît comme un outil de limitation. La formule de Michel Rocard selon laquelle "la France ne peut pas accueillir toute la misère du monde " - régulièrement adjointe de l'hypothétique " mais elle doit en prendre sa part » - est ainsi un leitmotiv des débats publics sur les marchés informels, où domine l'idée que cette " misère du monde " doit être gérée avec parcimonie. Les réponses locales mises en place à Paris sont nettement insuffisantes, puisque les dispositifs élaborés par les pouvoirs municipaux sont de taille limitée et très inférieure au nombre de vendeurs présents sur les marchés informels. Les dispositifs gestionnaires parcellaires contournent certains enjeux de conflits, mais suscitent de nouveaux mécontentements et produisent des lieux aux statuts pluriels et précaires.

A la Porte de Montmartre, les rivalités commerciales ont été détournées par la définition du Carré des biffins comme " dispositif expérimental d'action sociale » et sa gestion par l'association Aurore, faisant office de placier. Mais la répression des vendeurs exclus de ce Carré provoque des divisions au sein de la mobilisation (Milliot, 2010). Les riverains reconnaissent en tant que biffins les vendeurs du Carré, mais continuent à se plaindre de la présence des "vendeurs à la sauvette " qui s'installent sur l'avenue de la Porte de Montmartre et le mail Binet. "Ils n'ont pas résolu un problème, ils en ont créé un ", aime à dire Nicolas, biffin à Belleville. Le Carré constitue "une zone grise de droit ", selon l'adjoint communiste au maire du 18e arrondissement chargé de sa mise en place ; le dispositif revient par exemple à autoriser le travail des retraités. L'interposition de l'association Aurore permet à l'action des pouvoirs municipaux de s'inscrire en marge du droit pour gérer partiellement ces pratiques marchandes informelles des espaces publics. Mais "le travail des salariés d'Aurore est éprouvant " (Milliot, 2010 : 74). Dans l'entre-deux d'une gestion de l'espace public par intermédiaires interposés, la sous-traitance de l'action sociale à des associations fait appel à l'engagement des travailleurs sociaux pour colmater les brèches, tandis que le statut d' "expérimentation », depuis cinq ans, maintient le dispositif dans la précarité. Dans le 14e arrondissement, la tentative de contenir la vente informelle au sein du marché aux Puces témoigne d'une volonté de limiter les conflits autour de l'occupation de l'espace public par les vendeurs, en gérant l'informalité par l'intermédiaire de l'entreprise gestionnaire du marché aux Puces. La conflictualité se joue alors entre biffins et commerçants des Puces. Enfin, dans le 20e arrondissement, le projet de ressourcerie a en commun avec le Carré des biffins de faire intervenir une association d'action sociale. Le flou institutionnel et juridique des délégations de service public aux associations ${ }^{23}$ permet ainsi aux pouvoirs municipaux de bricoler des tentatives de contournement des tensions locales.

L'appel à des tiers associatifs s'inscrit dans le même contexte de promotion du local comme échelle de gestion territoriale qui a accompagné la valorisation du "cadre de vie » (Oblet, 2005 : 168-169). Les

23 " Le procédé associatif fait l'objet de mises au point fréquentes de la part des juridictions administratives et financières, du fait de l'emploi de fonds publics, voire de prérogatives publiques, qui peuvent échapper dans les faits aux règles auxquelles sont assujetties les collectivités locales » (Vital-Durand, 2011, p. 120). 
pouvoirs municipaux, se faisant gestionnaires par intermédiaires de l'action sociale, ont recours à des associations qui " se voient peu à peu chargées de gérer nombre d'aspects de la vie locale dont l'Etat cherche à se défaire " (Brunet et al., 1994 : 47). Les élus municipaux mettent en outre en concurrence les associations et les dispositifs gestionnaires élaborés. En confiant le projet de ressourcerie à I'association Emmaüs Coup de main, la municipalité du 20e arrondissement s'assure d'une rupture avec le Carré des biffins, installé dans l'espace public, et géré par l'association Aurore, dont les salariés soutiennent les revendications des biffins en matière d'ouverture de nouveaux espaces de vente. La précarisation de l'action sociale publique se fait donc dans le cadre d'une fragmentation gestionnaire. Or, celle-ci met à distance les biffins en fragilisant l'association Sauve qui peut par la multiplication des intermédiaires et des conflits politico-associatifs autour de l'accès à la gestion des alternatives mises en place pour les biffins. L'interposition d'un tiers associatif a ainsi contribué au délitement de l'espace intermédiaire de la mobilisation des biffins à la Porte de Montmartre en limitant leur accès aux instances de la prise de décision municipale (Milliot, 2010 : 66). L'intervention d'associations qui accèdent à des fonctions de gestion urbaine contribue à l'accroissement de la distance socio-politique entre les édiles métropolitains et les vendeurs de rue rejetés des espaces péricentraux qu'ils investissent comme ressource de survie. Elle participe à la marginalisation socio-politique de ces derniers en instaurant de nouvelles divisions au sein des marchés informels, à l'instar de ce qui se joue dans les pays du Sud autour de l'intervention des ONG (Canovas, 2008 : 159-160).

\section{Conclusion}

Les pouvoirs municipaux auxquels la décentralisation a conféré des compétences élargies, se trouvent donc confrontés à Paris à des "territoires de l'informel " (Steck, 2003) qui occupent les interstices du contrôle des espaces publics dans des quartiers péricentraux de la métropole. L'extension des marchés informels soulève des controverses autour des normes d'usage des espaces publics. Ces espaces de l'entre-deux sont en effet perçus par les responsables municipaux et certains citadins comme des menaces de submersion et de subversion des normes non seulement spatiales mais aussi socioéconomiques associées au modèle urbain occidental. Les enjeux en termes de propreté et de sécurité font écho à la menace d'une précarité rendue perceptible par ces marchés. Saisissant cette portée subversive, des partis politiques s'inscrivant dans le courant de la gauche alternative investissent ces espaces de l'entre-deux pour y défendre la possibilité d'une alternative gestionnaire construite à l'échelle locale. Ils mettent en avant cette économie de survie urbaine comme une forme de résistance à une situation de crise, alors que les municipalités socialistes tentent de défendre le " cadre de vie " urbain par des politiques répressives. La promotion du local comme échelle de l'alternative constitue ainsi un " outil de subversion politique » (Lefebvre, 2011 : 79), déjà employé par le Parti socialiste dans les années 1970 face à l'étatisme gaulliste, et qui s'inscrit dans le cadre de rivalités politico-territoriales autour de l'accès au gouvernement urbain. Mais l'espace intermédiaire de redéfinition des normes ouvert par la mobilisation des biffins se heurte aux "travers du localisme (...), querelle de clocher, clientélisme, populisme, démagogie même " (Vieillard-Baron, 2006 : 14). Les "solutions locales" prônées par la mobilisation des biffins se trouvent en effet confrontées aux enjeux supra-locaux de l'extension des marchés informels, et prises dans les contradictions de la politique locale. Elles aboutissent alors à la multiplication de situations d'entre-deux gestionnaires.

Les dynamiques métropolitaines de concentration urbaine et d'exacerbation des inégalités sociales, en perturbant les anciennes tolérances locales, font passer le traitement de ces activités de la tolérance 
informelle au plan institutionnel. Alors que la pauvreté rendue perceptible par ces marchés remet frontalement en cause le modèle occidental et les valeurs de justice sociale qui lui sont associées, les pouvoirs publics démultiplient les situations d'entre-deux en tentant de contenir l'extension de ces marchés. Ils ne normalisent pas plus la situation des vendeurs informels qu'ils n'éradiquent ces marchés ; au contraire, l'intervention des pouvoirs locaux s'accompagne de la production de nouvelles situations d'entre-deux spatiales et institutionnelles. Les dispositifs de gestion locale mis en place sous la pression de la mobilisation des biffins donnent moins lieu à l'émergence d'un nouveau modèle qu'ils ne traduisent une complexification et une opacification de la gouvernance urbaine, renforçant la distance entre édiles métropolitains et laissés-pour-compte de la métropolisation. Si le local semble d'abord permettre, dans le 18e arrondissement, le passage de l'entre-deux à l'alternative par le contournement des débats sur l'informel, il apparaît ensuite comme un outil de cantonnement. La délégation des compétences gestionnaires à des associations appelées par les responsables municipaux à s'immiscer dans la gestion urbaine marginalise la place des biffins dans les débats publics. Elle traduit le désengagement des pouvoirs publics et accompagne la fragmentation de l'action des pouvoirs publics. L'alternative est alors réduite à des initiatives parcellaires bien éloignées d'un rééquilibrage des rapports de domination englobants; la vulnérabilité des vendeurs informels, malgré les revendications autour du "droit à la ville » des biffins portées par des mouvements alternatifs, limite leur capacité à faire valoir leurs intérêts dans l'espace public.

\section{Bibliographie}

A.D.I.E. (2008), Etude-action sur les biffins. Chiffonniers, récupérateurs, vendeurs de la Porte Montmartre. Volets social et économique, accessible en ligne sur le site de I'ADIE, http://www.adie.org/sites/default/files/links/Biffins_08.08.pdf, 45 p.

BACQUE M.-H., SINTOMER Y. (2011), La démocratie participative. Histoire et généalogie, Paris, La Découverte, $288 \mathrm{p}$.

BACQUE M.-H. (2006), "En attendant la gentrification : discours et politiques à la Goutte d'Or (19822000)", Sociétés contemporaines, 2006/3, pp. 63-83.

BRUNET R., FERRAS R., THERY H. (1993), Les mots de la géographie. Dictionnaire critique, Paris, Reclus, La Documentation française, 3e édition, $518 \mathrm{p}$.

CANOVAS J. (2008), Nouveaux mouvements sociaux et néolibéralisme en Amérique latine. Des alternatives à un système globalisé ?, Paris, L'Harmattan, 188 p.

CHABROL M. (2012), De nouvelles formes de gentrification ? Dynamiques résidentielles et commerciales dans le quartier de Château-Rouge (Paris), Thèse de doctorat, Université de Poitiers, 404

p.

CHARPY M. (2011), "L'apprentissage du vide. Commerces populaires et espace public à Paris dans la première moitié du XIXe siècle", Espaces et Sociétés, 2011/1-2, n¹44-145, pp. 15-35.

CHOAY F. (1994), "Six thèses en guise de contribution à une réflexion sur les échelles d'aménagement et le destin des villes", in BERQUE A., La maîtrise de la ville. Urbanité française, urbanité nippone, Paris, Editions de l'EHESS, pp. 221-227.

CLERVAL A. (2013), Paris sans le peuple. La gentrification de la capitale, Paris, La Découverte, 280 p.

COSTE L. (1994), L'étranger sous terre. Commerçants et vendeurs à la sauvette du métro parisien, Paris, L'Harmattan, $186 \mathrm{p}$.

FAYMAN S. (2011), "La Bellevilleuse ou la mise en oeuvre du droit à la ville", in DEBOULET A., DE VILLANOVA R., Belleville, quartier populaire ?, Paris, Créaphis Editions, pp. 79-92. 
GERVAIS-LAMBONY (1994), De Lomé à Harare, le fait citadin. Images et pratiques des villes africaines, Paris, Karthala, $472 \mathrm{p}$.

GERVAIS-LAMBONY P., LANDY F. (dir.), 2007, On dirait le Sud..., Autrepart, 41, 257 p.

HARVEY D. (2012), Paris, capitale de la modernité, Paris, Les Prairies Ordinaires, 529 p.

HARVEY D. (2011), Le capitalisme contre le droit à la ville. Néolibéralisme, urbanisation, résistances, Editions Amsterdam, Paris, $93 \mathrm{p}$.

HATZFELD H., HATZFELD M., RINGART N. (1998), Quand la marge est créatrice. Les interstices urbains initiateurs d'emploi, La Tour d'Aigues, Editions de l'Aube, 155 p.

JACQUOT S. (2007), "Pour une définition relationnelle des termes Nord et Sud : Gênes et Valparaiso", Autrepart, 41, pp. 181-194.

LEFEBVRE H. (2009, 1968), Le droit à la ville, Paris, Economica-Anthropos, 135 p.

LEFEBVRE R. (2011), "Retour sur les années 1970. Le Parti socialiste, l'autogestion et la démocratie locale", in BACQUE M.-H., SINTOMER Y., La démocratie participative. Histoire et généalogie, Paris, La Découverte, pp. 65-81.

LYNCH K. (1960), L'image de la cité, Paris, Dunod, 222 p.

MILLIOT V. (2010), "La ville informelle et le travail des marges", in MILLIOT V., TASTEVIN Y.-P., Les archipels de la Goutte d'Or. Analyse anthropologique d'une métropolisation par le bas, Rapport de recherche pour le programme "Culture et territoires en Ile-de-France", pp. 7-89.

MILLIOT V. (2011), "Les biffins, ou l'organisation de la misère ?", Territoires, octobre 2011, pp. 46-49.

OBLET T. (2005), Gouverner la ville. Les voies urbaines de la démocratie moderne, P.U.F., 306 p.

PERALDI M. (2002), La fin des norias ?, Maisonneuve et Larose, Paris, 495 p.

RULLAC S., WEISS C., et al., 2012, Réalisation d'une cartographie localisant les lieux de vente de récupérateurs-vendeurs sur le territoire francilien, Marché public $n^{\circ} 1100292$ lot $n^{\circ} 1$, Association Aurore/BUC ressources/CERA, $74 \mathrm{p}$.

RULLAC S., BAZIN H., et al., 2012, Etude qualitative portant sur les conditions de vie des biffins en llede-France, Marché public n¹10 0292 lot n², Association Aurore/BUC Ressources/CERA, $126 \mathrm{p}$.

SASSEN S. (1998), Globalization and its Discontents. Essays on the new mobility of people and money, New York, The New Press, 253 p.

SERREAU C. (2010), Solutions locales pour un désordre global, Paris, Acte Sud, 256 p.

STECK J.-F. (2003), Territoires de l'informel : les petites activités de rue, le politique et la ville à Abidjan, Thèse de doctorat, Université de Paris 10-Nanterre, $600 \mathrm{p}$.

RAOULX B. (2006), "East Side, West Side. L'expérience d'une recherche et d'un film documentaire avec les "fouilleurs de poubelles" de Vancouver", Etudes canadiennes/Canadian Studies, 2006/60, pp. 151180.

RAOULX B. (1999), "La pratique de l'espace urbain des populations marginales : l'exemple des "binners" de Vancouver", Espace, populations, sociétés, 1999/2, pp. 353-357.

RAOULX B. (2006), "De l'espace-miroir à l'espace-écran : un effet médiatique ?" in SECHET R., VESCHAMBRE V., Penser et faire la géographie sociale. Contributions à une épistémologie de la géographie sociale, Rennes, P.U.R., pp. 149-172.

TONNELAT (2003), Interstices urbains, Paris - New York. Entre contrôles et mobilités, quatre espaces résiduels de l'aménagement, Thèse de doctorat, Université de Paris 12/IUP - CUNY, 592 p.

VALLAT C., SEMMOUD N., MONNET J., LEFRANÇOIS D. (2008), "Constructions illégales, activités informelles, interstices urbains : la ville, indomptable territoire", Historiens et Géographes, 403, pp.113-123.

VIEILLARD-BARON H. (2006), "Le terrain et la proximité en question" in SECHET R., VESCHAMBRE V., 
Carnets de géographes, $\mathrm{n}^{\circ} 7$, juin 2014

Rubrique Carnets de recherche

Penser et faire la géographie sociale. Contributions à une épistémologie de la géographie sociale, Rennes, P.U.R., pp. 133-148.

VITAL-DURAND E. (2011), Les collectivités territoriales en France, Paris, Hachette Supérieur, 157 p. 